\title{
Suggestions for safety and protection control in Department of Nuclear Medicine during the outbreak of COVID-19
}

\author{
Xiao Zhang ${ }^{1,2} \cdot$ Fuqiang Shao ${ }^{1,2} \cdot$ Xiaoli Lan ${ }^{1,2}$ \\ Received: 3 March 2020 / Accepted: 18 March 2020 / Published online: 25 March 2020 \\ (C) Springer-Verlag GmbH Germany, part of Springer Nature 2020
}

Dear Sir,

Since December 2019, a novel coronavirus disease named COVID-19 broke out in Wuhan [1], Hubei province, China, and then spread throughout the country. At present, growing cases have been found in other countries with a rapid growth rate, and the world moves closer toward worldwide spread of the virus [2]. European Centre for Disease Prevention and Control (EDCD) had raised the risk infection level of COVID-19 from medium to high for its severe situation [3]. The virus spread rapidly from person to person through the droplets of respiratory and close contact, and everyone can be infected [4]. The common clinical symptoms of COVID-19 include fever, cough, and myalgia or fatigue. Old men with comorbidities are more likely to have severe or even fatal respiratory diseases, such as acute respiratory distress syndrome [4]. In our department, four highly suspected patients with COVID-19 underwent [18F]-FDG PET/CT in January 2020 [5]. PET and SPECT are routine examinations for clinical diagnosis of various diseases and appropriate protective measures should be carried out during this epidemic.

Organized by Chinese Society of Nuclear Medicine and Editorial Board of Chinese Journal of Nuclear Medicine and Molecular Imaging, expert consensus on the safety prevention for nuclear medicine diagnosis and treatment during the outbreak of COVID-19 (1st edition) has been published in China [6]. The aim was to effectively control the source of infection, cut off the transmission route, protect the susceptible

This article is part of the Topical Collection on Letter to the Editor

Xiaoli Lan

xiaoli_lan@hust.edu.cn; 1x1730724@hotmail.com

1 Department of Nuclear Medicine, Union Hospital, Tongji Medical College, Huazhong University of Science and Technology, No. 1277 Jiefang Ave, Wuhan 430022, China

2 Hubei Province Key Laboratory of Molecular Imaging, Wuhan 430022, China population, and ensure medical quality and safety. At the center of epidemic area, Wuhan, China, some preventives measures have been taken in our department.

It is important to recognize and classify all patients. Patients with confirmed or suspected diagnosis of COVID19 should be invited to postpone the radiopharmaceutical imaging and treatment. If radiopharmaceutical imaging is really necessary for them, it is recommended to arrange them in an isolated room equipped with a high-efficiency particulate air filter. Furthermore, the patient should be scheduled as the last one of the day, in order to avoid cross infection. All patients and accompanying persons must wear a protective mask during the entire examination. It is important to try to shorten the examination time and limit the patients' activities.

During the outbreak of COVID-19, all medical personnel should wear the medical uniform, cap, and mask. Hand hygiene is very important. For the medical staffs who inject the radiopharmaceuticals to the patients with confirmed or suspected diagnosis of COVID-19, safety goggles or face shield, isolation gown, disposable latex gloves, and shoe covers are needed. If special treatments (such as sputum aspiration and specimen collection) are performed in an emergency, upgraded protections for respiratory and eyes are necessary [7]. The operating table, instruments (SPECT, SPECT/CT, $\mathrm{PET} / \mathrm{CT}$ and PET/MR), and the environment must be cleaned and disinfected promptly.

In Wuhan, the center of the epidemic area, particular attention is paid to avoid the exposure to coronavirus in the Department of Nuclear Medicine. In our department, patients are required to perform chest CT and RT-PCR test for SARS-CoV-2, to exclude the diagnosis of COVID-19 before radiopharmaceutical imaging. In non-endemic areas, it is also necessary to recognize and classify all patients, verify the history and clinical symptoms of every patient. We hope our experience could help to safely conduct the nuclear medicine work, reduce the risk of disease transmission, and guarantee the medical quality and safety. 


\section{Compliance with ethical standards}

Human and animal rights This article does not contain any studies with human participants or animals performed by any of the authors.

Conflict of interest The authors declare that they have no conflict of interest.

\section{References}

1. Coronavirus latest: WHO officially names disease COVID-19. Nature News. 2020. https://www.nature.com/articles/d41586-02000154-w. Accessed 11 Feb 2020.

2. Centers for Disease Control and Prevention. Coronavirus Disease 2019 (COVID-19): Coronavirus Disease 2019 (COVID-19) Situation Summary. 2020. https:/www.cdc.gov/coronavirus/2019nCoV/summary.html. Accessed 29 Feb 2020.

3. Rapid risk assessment: Outbreak of novel coronavirus disease 2019 (COVID-19): increased transmission globally - fifth update. European Centre for Disease Prevention and Control. 2020. https:// www.ecdc.europa.eu/en/publications-data/rapid-risk-assessmentoutbreak-novel-coronavirus-disease-2019-covid-19-increased. Accessed 20 Feb 2020 .
4. Huang C, Wang Y, Li X, Ren L, Zhao L, Fan G, et al. Clinical features of patients infected with 2019 novel coronavirus in Wuhan, China. Lancet. 2020;395:497-506. https://doi.org/10.1016/ S0140-6736(20)30183-5.

5. Qin C, Liu F, Yen T, Lan X. ${ }^{18}$ F-FDG PET/CT findings of COVID19: a series of four highly suspected cases. Eur J Nucl Med Mol Imaging. 2020. https://doi.org/10.1007/s00259-020-04734-w.

6. Chinese Society of Nuclear Medicine and Editorial Board of Chinese Journal of Nuclear Medicine and Molecular Imaging. Expert consensus on the safety prevention and control of nuclear medicine diagnosis and treatment during the outbreak of COVID-19 (1st edition). Chin Nucl Med Mol Imaging, 2020;40. DOI: https://doi.org/10. 3760/cma.j.cn321828-20200222-00062.

7. Centers for Disease Control and Prevention. Coronavirus Disease 2019 (COVID-19): Interim Infection Prevention and Control Recommendations for Patients with Confirmed Coronavirus Disease 2019 (COVID-19) or Persons Under Investigation for COVID-19 in Healthcare Settings. 2020. https://www.cdc.gov/ coronavirus/2019-ncov/infection-control/control-recommendations. html. Accessed 21 Feb 2020.

Publisher's note Springer Nature remains neutral with regard to jurisdictional claims in published maps and institutional affiliations. 